\title{
Solving the first Painleve equation using the Sinc-Galerkin method
}

\author{
Mehrnoosh Eskandarnezhad*, Sedaghat Shahmorad, Ali Salimi Shamloo \\ Student of M.Sc in Applied Mathematics, Shabestar Branch, Islamic Azad \\ Department of Mathematics, Tabriz University, Tabriz, Iran \\ Department of Mathematics, Shabestar Branch, Islamic Azad University, Shabestar, Iran \\ *Corresponding author E-mail: m_eskandarnezhad@yahoo.com
}

\begin{abstract}
The main goal of this paper is to solve the first Painleve equation using the sinc-Galerkin method. In order to obtain this goal, we first describe briefly the sinc-Gelrkin method, and then we apply it to the first Painleve equation. At last, the numerical result is shown to demonstrate the power and accuracy of the method.
\end{abstract}

Keywords: The first Painleve Equation, Sinc-Galerkin Method

\section{Introduction}

The Painleve equations were first found by Painleve and his collaborators more than a century ago when Painleve and his school began an investigation of the nonlinear second order ODEs class. They wanted to solve two different problems: to classify second-order differential equations of a certain form on the basis of their possible singularities of solutions, and to identify second-order differential equations which define new functions. The latter problem was formulated by Fuchs and Poincare in 1884. However, Fuchs and Poincare did not find any new functions because they considered the first-order ODE class. Painleve and his school found 50 canonical classes of equations whose solutions have no movable critical points. Furthermore, they also showed that among 50 equations there are exactly six secondorder ODEs that define new functions. At the present time these new functions are called Painleve transcendents; and equations with general solutions in the form of these transcendents are called Painleve equations. These six Painleve equations were first discovered from strictly mathematical investigations but these equations have recently appeared in several physical applications [1]. Many situations in science and engineering reduce to Painleve equations. Its applications include plasma physics, waves, optics and mechanics. The main goal of this research, here, is to use sinc methods for solving the first Painleve equation. The Painleve equations, first considered by Painleve, are defined as follows:

$$
u^{\prime \prime}(x)=f\left(x, u, u^{\prime}\right),
$$

where $f\left(x, u, u^{\prime}\right)$ is a rational function in $u$ and $u^{\prime}$, while $f$ is analytic in $x$. In this paper, the first Painleve equation will be considered in the following form,

$$
\begin{aligned}
& u^{\prime \prime}(x)=6 u^{2}(x)+x, x \in[0,1], \\
& u(0)=1, u^{\prime}(0)=0 .
\end{aligned}
$$

Several numerical methods $[6,8]$ for approximating the solutions of the first Painleve equation have been implemented. In this study, a numerical method for solving the first Painleve equation based on the Sinc-Galerkin approximations is described. Most numerical approximation techniques are based on the use of polynomials. Polynomial approximation formulas are applicable for approximation over finite intervals, or finite contours. In more than one dimension, polynomials are the basis for finite element approximation. But, Sinc approximation formulas are applicable over finite, 
semi-infinite, infinite intervals, or over contours. In more than one dimension, curvilinear regions are also readily dealt with. In addition, Sinc methods readily handle infinite regions. Polynomials and splines seem to have found their home in Sobolev spaces defined over bounded domains, whereas Sinc functions live more naturally in spaces of analytic functions defined over bounded or unbounded domains. Finite element methods were developed first, and this is perhaps why they are by far the most popular, whereas Sinc methods came later, and are much less widely in use. Sinc and finite element methods both enjoy simplicity and flexibility, although this is less well known for Sinc methods. The derived system of algebraic equations is usually more sparse for finite difference and finite element methods than for Sinc methods. In spite of this, if accuracy is desired in the solution of a differential or integral equation, the complexity of solving a differential equation problem via a finite difference or finite element method is usually far larger than the corresponding complexity for Sinc methods [16].

This paper is organized as follows: The sinc-Galerkin method is described in Section 2. In Section 3, the sinc-Galerkin method is developed for solving the first Painleve equation. Finally, numerical result is presented in Section 4.

\section{The Sinc-Galerkin method}

In this section, we will review Sinc function properties, Sinc quadrature rule, and the Sinc method. These are discussed thoroughly in [11] and [14]. Sinc methods for differential equations were originally introduced by Stenger in [15]. Since then they have become increasingly popular and have been well studied. These methods have been used for solving many differential and integral equations $[2,3,4,5,7,9,10,11,13,15,17,18]$. Definitions, notation, and properties of Sinc functions and composite Sinc functions can be found in references [7, 10].

Definition.1. [11] If $f$ is analytic in a domain $D$ and $z_{0} \in D$, then $f$ is called conformal at $z_{0}$ if $f^{\prime}\left(z_{0}\right) \neq 0$. If $f^{\prime}(z) \neq 0$ for all $z \in D$, then $f$ is called a conformal mapping of $D$. The Sinc function is defined on the whole real line by

$$
\operatorname{Sinc}(x)=\left\{\begin{array}{c}
\frac{\sin (\pi x)}{\pi x}: x \neq 0 \\
1: x=0
\end{array}\right.
$$

Definition.2. [11] Let $D$ be a domain in the $w=u+i v$ plane with boundary points $a \neq b$. Let $z=\varphi(w)$ be a one-toone conformal map of $D$ onto the infinite strip

$$
D_{S} \equiv\{z \in \mathbb{C}: z=x+i y:|y|<d\} \text {, }
$$

where $\varphi(a)=-\infty$ and $\varphi(b)=\infty$. Denote by $w=\psi(z)$ the inverse of the mapping $\varphi$ and let

$$
\Gamma \equiv\{w \in \mathbb{C}: w=\psi(x), x \in R\}=\psi(R) .
$$

Let $B(D)$ denote the class of functions analytic in $D$ which satisfy for some constant $a$ with $0 \leq a<1$,

$$
\int_{\psi(x+L)}|F(w) d w|=O\left(|x|^{a}\right) \text {, as } x \rightarrow \pm \infty
$$

where $L=\{i y:|y|<d\}$ and for $\gamma$ a simple closed contour in $D$

$$
N(F, D) \equiv \lim _{\gamma \rightarrow \partial D} \int_{\gamma}|F(w) d w|<\infty
$$

Now, we want to present the interpolation and quadrature rule based on the Sinc functions. The following theorem is the interpolation theorem for $F$. 
Theorem.2.1. Let $\varphi^{\prime} F \in B(D)$ and $h>0$. Let $\varphi$ be a one-to-one conformal map of the domain $D$ onto $D_{s}$. Let $\psi=\varphi^{-1}, w_{k}=\psi(k h)$ and for $\Gamma=\psi(R)$. Then for all,

$$
\varepsilon\left(\varphi^{\prime} F\right)(\xi)=F(\xi)-\sum_{k=-\infty}^{\infty} F\left(w_{k}\right) \operatorname{Sinc}\left(\frac{\varphi(\xi)-k h}{h}\right),
$$

is bounded by

$$
\left\|\varepsilon\left(\varphi^{\prime} F\right)\right\|_{\infty} \leq \frac{N\left(\varphi^{\prime} F, D\right)}{2 \pi d \sinh (\pi d / h)}
$$

Further assume that there are positive constants $\alpha, \beta$, and $C$ so that

$$
|F(\xi)| \leq C\left\{\begin{array}{l}
\exp (-\alpha|\varphi(\xi)|), \xi \in \Gamma_{a} \\
\exp (-\beta|\varphi(\xi)|), \xi \in \Gamma_{b}
\end{array}\right.
$$

where

$$
\Gamma_{a}=\{\xi \in \Gamma: \varphi(\xi)=x \in(-\infty, 0)\}, \Gamma_{b}=\{\xi \in \Gamma: \varphi(\xi)=x \in[0, \infty)\},
$$

If the selections

$$
N=\left[\left|\frac{\alpha}{\beta} M+1\right|\right], h=\left(\frac{\pi d}{\alpha M}\right)^{1 / 2} \leq \frac{2 \pi d}{\ln (2)},
$$

are made, then for all $\xi \in \Gamma$ the error

$$
\varepsilon_{M, N}\left(\varphi^{\prime} F\right)(\xi) \equiv F(\xi)-\sum_{k=-M}^{N} F\left(w_{k}\right) \operatorname{Sinc}\left(\frac{\varphi(\xi)-k h}{h}\right)
$$

is bounded by

$$
\left\|\varepsilon_{M, N}\left(\varphi^{\prime} F\right)\right\|_{\infty} \leq K \sqrt{M} \exp \left(-(\pi d \alpha M)^{1 / 2}\right)
$$

and $K$ is a constant depending on $F, d, \varphi$, and $D$.

Proof: Refer to [11]. Under the assumptions of this theorem, we can state the quadrature rule [11, 12] which approximates the integral, i.e.,

$$
\eta_{M, N}(F) \equiv \int_{\Gamma} F(\xi) d \xi-h \sum_{k=-M}^{N} \frac{F\left(w_{k}\right)}{\varphi^{\prime}\left(w_{k}\right)},
$$

and this is bounded by

$$
\left\|\eta_{M, N}\left(\varphi^{\prime} F\right)\right\|_{\infty} \leq G \exp \left(-(\pi d \alpha M)^{1 / 2}\right)
$$

and $G$ is a constant depending on $F, d, \varphi$ and $D$.

Now, we briefly discuss the Sinc-Galerkin method. For the Sinc method, the basis functions on the $\Gamma=(a, b)$ are derived from the composite translated Sinc functions

$$
S_{j}(x)=S(j, h) \circ \varphi(x)=\operatorname{Sinc}\left(\frac{\varphi(x)-j h}{h}\right),
$$


where $h>0, j$ is an integer, that is, these functions are the basis functions, and the conformal map is given by

$$
\varphi(x)=\ln \frac{x-a}{b-x},
$$

The Sinc nodes $x_{k}$ are chosen so that

$$
x_{k} \equiv \psi(k h)=\varphi^{-1}(k h)=\frac{a+b e^{k h}}{e^{k h}+1} .
$$

The Sinc-Galerkin method converges exponentially, even in the presence of boundary singularities as shown in [11, 14]. The approximate solution for $u(x)$ is represented by the formula

$$
u_{m}(x)=\sum_{j=-M}^{N} u_{j} S_{j}(x), m=M+N+1 .
$$

The unknown coefficients $u_{j}$ in equation (2.18) are determined by orthogonalizing the residual with respect to the basis functions, i.e., The orthogonalization of the residual in the Sinc-Galerkin method for a differential equation of the form

$$
L u=f, o n(a, b)
$$

where $L$ is a differential operator, can be treated as follows:

$$
<L u_{m}-f, S_{k}>=0,-M \leq k \leq N .
$$

The choice of the inner product that is used in equation (2.20), along with the choice of basis functions, determines the properties of the approximation method. The inner product that is used for the Sinc-Galerkin method is defined by

$$
<f, g>=\int_{a}^{b} f(x) g(x) w(x) d x .
$$

Here is a weight function that is chosen depending on the boundary conditions, the domain, and the differential equation. For our problem, it is convenient to take

$$
w(x)=\frac{1}{\varphi^{\prime}(x)} .
$$

A broad discussion of the choice of weight function can be found in $[11,14,16]$. The most direct development of the discrete system for (2.19) is obtained by substituting (2.18) into (2.20). This approach, however, obscures the analysis that is necessary for applying the Sinc quadrature formulas. Instead of directly applying the quadrature rule to the inner products, the Sinc-Galerkin method proceeds as follows: Let us present it in the following section as it will be appllied to the first painleve equation.

\section{Application of Sinc-Galerkin method to the first order Painleve equation}

we can write the equation (1.2) as

$$
N u(x)=f(x)
$$

Where $N$ and $f$ are as following 


$$
N u(x)=u "(x)-6 u^{2}(x), f(x)=x .
$$

The residual for equation (3.1) is

$$
R_{m}(x)=\left(N u_{m}-f\right)(x),
$$

The orthogonalization of the residual in the above equation takes the form

$$
<N u_{m}-f, S(j, h) \circ \varphi>=0,
$$

This orthogonalization of the residual is more conveniently treated by first considering the inner products in

$$
<N u-f, S(j, h) \circ \varphi>=0
$$

By writing in the integral form, we get,

$$
\begin{array}{r}
\int_{0}^{1} u "(x) S(j, h) \circ \varphi(x) w(x) d x-\int_{0}^{1} u^{2}(x) S(j, h) \circ \varphi(x) w(x) d x \\
-\int_{0}^{1} f(x) S(j, h) \circ \varphi(x) w(x) d x=0 .
\end{array}
$$

Using the quadrature rule to approximate each of the these integrals leads to the same discrete system as the system obtained from (3.4). In each inner product arising from (3.5) integrate by parts those terms containing derivatives of $u$ to remove the derivatives from $u$. For example, the inner product containing the second derivative of $u$ reads

$$
\begin{aligned}
<u "(x), S_{j}(x) & >=\int_{a}^{b} u "(x)[S(j, h) \circ \varphi(x)] w(x) d x \\
& =B_{T}+\int_{a}^{b} u(x)[S(j, h) \circ \varphi(x) w(x)] " d x .
\end{aligned}
$$

Where

$$
B_{T}=\left.\left(u^{\prime}(x)[S(j, h) \circ \varphi(x) w(x)]\right)\right|_{a} ^{b}-\left.\left(u(x)[S(j, h) \circ \varphi(x) w(x)]^{\prime}\right)\right|_{a} ^{b} .
$$

The reason for the integration by parts is twofold. Firstly, the selection of the weight $w$ can be based on criteria such as forcing the boundary term $B_{T}$ to vanish. Secondly, $u_{m}$ can for suitable $u_{j}, x_{j}=\varphi^{-1}(j h)$ provide a good approximation of $u$ [11]. Now, we are in a position to apply the approximation to formulate the discrete Sinc-Galerkin system for the problem (4.1). The Galerkin equations for $j=-M, \ldots, 0, \ldots, N$ are

$$
\begin{aligned}
0=<N u-f, S(j, h) \circ \varphi(x)>=-<u "(x), S(j, h) \circ \varphi(x)> \\
-<6 u^{2}(x), S(j, h) \circ \varphi(x)>-<f, S(j, h) \circ \varphi(x)>,
\end{aligned}
$$

Approximating the inner products using quadrature rule and replacing $u\left(x_{k}\right)$ by $u_{k}$ yield the discrete Sinc system

$$
\begin{aligned}
\sum_{k=-M}^{N}[ & \left.\frac{-1}{h^{2}} \delta_{j k}^{(2)} \varphi\left(x_{k}\right) w\left(x_{k}\right)-\frac{1}{h} \delta_{j k}^{(1)}\left(\frac{\varphi^{\prime \prime}\left(x_{k}\right) w\left(x_{k}\right)}{\varphi^{\prime}\left(x_{k}\right)}+2 w^{\prime}\left(x_{k}\right)\right)\right] u_{k} \\
& -\frac{w^{\prime \prime}\left(x_{j}\right)}{\varphi^{\prime}\left(x_{j}\right)} u_{j}+6 \frac{w\left(x_{j}\right)}{\varphi^{\prime}\left(x_{j}\right)} u_{j}^{2}=\frac{f\left(x_{j}\right) w\left(x_{j}\right)}{\varphi^{\prime}\left(x_{j}\right)}, j=-M, \ldots, 0, \ldots, N .
\end{aligned}
$$

where $\delta_{j k}^{(2)}$ and $\delta_{j k}^{(1)}$ are that introduced in the following theorem:

Theorem.1. Let $\varphi$ be a conformal one-to-one map of the simply connected domain $D$ onto $D_{s}$. Then 


$$
\begin{aligned}
& \delta_{j k}^{(0)}=\left.S(j, h) \circ \varphi(x)\right|_{x=x_{k}}=\left\{\begin{array}{lll}
1 & : & j=k \\
0 & : & j \neq k,
\end{array}\right. \\
& \delta_{j k}^{(1)}=\left.h \frac{d}{d \varphi} S(j, h) \circ \varphi(x)\right|_{x=x_{k}}=\left\{\begin{array}{cc}
0 & : j=k \\
\frac{(-1)^{k-j}}{k-j}: & j \neq k,
\end{array}\right.
\end{aligned}
$$

and

$$
\delta_{j k}^{(2)}=\left.h^{2} \frac{d^{2}}{d \varphi^{2}} S(j, h) \circ \varphi(x)\right|_{x=x_{k}}= \begin{cases}-\frac{\pi^{2}}{3}: & j=k \\ -\frac{2(-1)^{k-j}}{(k-j)^{2}}: & j \neq k,\end{cases}
$$

Proof: Refer to [11]. This system (2.14) is identical to that generated from orthogonalizing the residual via $<N u_{m}-f, S(j, h) \circ \varphi(x)>=0[11]$.

\section{Numerical result}

In this section, we want to test the mentioned method through the test problems. Hence, three numerical example will be considered and their approximate and exact solutions are compared and the results are shown in the related tables.

Example1: In order that $u_{m}$ satisfies the boundary conditions [11], it must be selected as

$$
u_{m}(x)=1+\sum_{j=-M}^{N} u_{j} S_{j}(x) .
$$

Now, by solving the system of nonlinear equations (3.10), we get the following result shown in the table1.

Table 1: Numerical results

\begin{tabular}{cccc}
\hline$x_{j}$ & The values of $u_{m}\left(x_{j}\right)$ & $x_{j}$ & The values of $u\left(x_{j}\right)$ \\
\hline 0.0 & 1 & 0.6 & 0.7 \\
0.1 & 1.03047 & 0.8 & 3.81083 \\
0.2 & 1.12626 & 0.9 & 5.56237 \\
0.3 & 1.30145 & 1.0 & 12.85161 \\
0.4 & 1.58238 & & \\
\hline
\end{tabular}

Example 2: Consider the following two-point boundary value problem [11]

$$
\begin{gathered}
-u^{\prime \prime}(x)-\frac{1}{6 x} u^{\prime}(x)+\frac{1}{x^{2}} u(x)=\frac{19}{6} \sqrt{x}, \quad 0<x<1, \\
u(0)=u(1)=0,
\end{gathered}
$$

The exact solution of the above problem is

$$
u(x)=x^{\frac{3}{2}}(1-x)
$$

In this example, we must choose 


$$
u_{m}(x)=\sum_{k=-M}^{N} u_{k} s(k, h) \circ \varphi(x), \quad m=-M, \ldots, 0, \ldots N,
$$

Where $S$ and $\varphi$ are as the same in preceding section. By a similar process in that section, one can get:

$$
\begin{gathered}
\sum_{k=-M}^{N}\left[\frac{-1}{h^{2}} \delta_{j k}^{(2)} \varphi^{\prime}\left(x_{k}\right) w\left(x_{k}\right)-\frac{1}{h} \delta_{j k}^{(1)}\left(\frac{\varphi^{\prime \prime}\left(x_{k}\right) w\left(x_{k}\right)}{\varphi^{\prime}\left(x_{k}\right)}+2 \varphi^{\prime}\left(x_{k}\right)\right)\right] u_{k}-\frac{w^{\prime \prime}\left(x_{j}\right)}{\varphi^{\prime}\left(x_{j}\right)} u_{j} \\
+\frac{1}{6} \sum_{k=-M}^{N} \frac{1}{h} \delta_{j k}^{(1)} \frac{1}{x_{k}} w\left(x_{k}\right) u_{k}+\frac{1}{6} \frac{(x w)^{\prime}\left(x_{j}\right)}{\varphi^{\prime}\left(x_{j}\right)} u_{j} \\
+\frac{w\left(x_{j}\right)}{x_{j}{ }^{2} \varphi^{\prime}\left(x_{j}\right)} u_{j}=\frac{19}{6} \frac{\sqrt{x_{j}} w\left(x_{j}\right)}{\varphi^{\prime}\left(x_{j}\right)}, \quad j=-M, \ldots, 0, \ldots, N .
\end{gathered}
$$

By solving this system of linear equations, we can find the approximate solution $u_{m}$. In table 2 the approximate and the exact solution of the above example are compared.

Table 2: Numerical Result For example 2

\begin{tabular}{cccc}
\hline $\mathrm{X}$ & The Approximate solution & The exact solution & Absolute error \\
\hline 0 & 0 & 0 & 0 \\
0.1 & 0.028460493 & 0.028460499 & $5.50 \mathrm{E}-09$ \\
0.2 & 0.071554161 & 0.071554175 & $1.43 \mathrm{E}-08$ \\
0.3 & 0.115021711 & 0.115021737 & $2.59 \mathrm{E}-08$ \\
0.4 & 0.151789288 & 0.151789328 & $3.96 \mathrm{E}-08$ \\
0.5 & 0.176776639 & 0.176776695 & $5.53 \mathrm{E}-08$ \\
0.6 & 0.185903128 & 0.185903201 & $7.26 \mathrm{E}-08$ \\
0.7 & 0.175698514 & 0.175698606 & $9.14 \mathrm{E}-08$ \\
0.8 & 0.143108239 & 0.143108351 & $1.12 \mathrm{E}-07$ \\
0.9 & 0.085381364 & 0.085381497 & $1.33 \mathrm{E}-07$ \\
1 & 0 & 0 & $0.00 \mathrm{E}+00$ \\
\hline
\end{tabular}

Example 3: [11] as the third example discussed in this paper, consider the problem

$$
\begin{gathered}
-u^{\prime \prime}(x)+\kappa u^{\prime}(x)=f(x), \quad 0<x<1, \\
u(0)=u(1)=0,
\end{gathered}
$$

Where $\kappa$ is a constant. In the case $f(x)=\kappa$, the exact solution of the problem is

$$
u(x)=x-\frac{e^{\kappa x}-1}{e^{\kappa}-1} .
$$

To solve the above problem using the Sinc-Galerkin method, one must choose

$$
u_{m}(x)=\sum_{k=-M}^{N} u_{k} s(k, h) \circ \varphi(x), \quad m=-M, \ldots, 0, \ldots N,
$$

Similarly, we can obtain

$$
\begin{aligned}
\sum_{k=-M}^{N}\left[\frac{-1}{h^{2}} \delta_{j k}^{(2)} \varphi^{\prime}\left(x_{k}\right) w\left(x_{k}\right)-\frac{1}{h} \delta_{j k}^{(1)}\left(\frac{\varphi^{\prime \prime}\left(x_{k}\right) w\left(x_{k}\right)}{\varphi^{\prime}\left(x_{k}\right)}+2 \varphi^{\prime}\left(x_{k}\right)\right)\right] u_{k}-\frac{w^{\prime \prime}\left(x_{j}\right)}{\varphi^{\prime}\left(x_{j}\right)} u_{j} \\
-\kappa \sum_{k=-M}^{N} \frac{1}{h} \delta_{j k}^{(1)} w\left(x_{k}\right) u_{k}-\kappa \frac{w\left(x_{j}\right)}{\varphi^{\prime}\left(x_{j}\right)} u_{j}=\kappa \frac{w\left(x_{j}\right)}{\varphi^{\prime}\left(x_{j}\right)}, \quad j=-M, \ldots, 0, \ldots, N .
\end{aligned}
$$


Solving this system of linear equations, one can calculate the approximate solution. The numerical result for this example is shown in the table 3.

Table 3: Numerical result for example 3 in the case $\mathrm{k}=10$

\begin{tabular}{cccc}
\hline $\mathrm{X}$ & The Approximate solution & The exact solution & Absolute error \\
\hline 0 & 0 & 0 & 0 \\
0.1 & 0.099895629 & 0.099921986 & $2.63 \mathrm{E}-05$ \\
0.2 & 0.199683538 & 0.199709924 & $2.63 \mathrm{E}-05$ \\
0.3 & 0.299106991 & 0.299133478 & $2.64 \mathrm{E}-05$ \\
0.4 & 0.397539588 & 0.397566537 & $2.69 \mathrm{E}-05$ \\
0.5 & 0.493279343 & 0.493307149 & $2.78 \mathrm{E}-05$ \\
0.6 & 0.581698768 & 0.581728931 & $3.01 \mathrm{E}-05$ \\
0.7 & 0.650219109 & 0.650256073 & $3.69 \mathrm{E}-05$ \\
0.8 & 0.664648939 & 0.664703974 & $5.50 \mathrm{E}-05$ \\
0.9 & 0.532045187 & 0.532149258 & $1.04 \mathrm{E}-04$ \\
1 & 0 & 0 & $0.00 \mathrm{E}+00$ \\
\hline
\end{tabular}

As was observed, the numerical results show that the Sinc-Galerkin solutions are good approximations for the exact solutions of the first Painleve equation. It is seen that the Sinc-Galerkin method is a powerful and accurate method for finding the solution of first Painleve equation. Moreover, it is straightforward and avoids the hectic work of calculations. The authors believe that the procedure as described in the present paper will considerably be of benefits to engineers and scientists working in this field.

\section{References}

[1] M. J. Ablowitz and P. A. Clarkson, Solitons, Nonlinear Evolution Equation and Inverse Scattering, Cambridge: Cambridge University Press, 1991.

[2] M. S. Akel, H. S. Hussein, Numerical treatment of solving singular integral equations by using Sinc approximations, Applied Mathematics and Computation, 218 (2011), pp.35653573.

[3] K. Al-Khaled, D. Kaya and M. A. Noor, Numerical comparison of methods for solving parabolic equations, Applied Mathematics and Computation, 157 (2004), pp. 735-743.

[4] M. T. Alquran and K. Al-Khaled, Approximations of Sturm-Liouville Eigenvalues Using Sinc-Galerkin and Diff erential Transform Methods, Appl. Appl. Math., Vol. 5, Issue 1 (June 2010), pp. 128 - 147.

[5] M. EL-Gamel, J. R. Cannon and A. I. Zayed, Sinc-Galerkin method for solving linear sixth-order boundary-value problems, MATHEMATICS OF COMPUTATION Volume 73, Number 247,(2003), pp. 1325-1343.

[6] B. Fornberg and J.A.C. Weideman, A numerical methodology for the Painleve equations, Journal of Computational Physics, 230 (2011), pp. 5957-5973.

[7] K. El-Kamel, Sinc numerical solution for solitons and solitary waves, J. Comput. Appl. Math., 130 (2001), pp. $283-292$.

[8] E. Hesameddini and S. Shekarpaz, Wavelet solutions of the first Painleve equation, 23rd International Conference of Jangjeon Mathematical Society, Shahid Chamran Uinvesity of Ahvaz, Ahvaz, Iran, (2010).

[9] K. Jalaei, M. Zarebnia and M. Mirzaee Chalaki, Development of the Sinc Method for Nonlinear Integro-Diff erential Eequations, Australian Journal of Basic and Applied Sci- ences, 4(11), (2010), pp. 5508-5515.

[10] J. Lund, Symmetrization of the Sinc-Galerkin method for boundary value problems, Math. Comp., 47 (1986), pp. $571-588$.

[11] J. Lund, K.L. Bowers, Sinc Methods for Quadrature and Diff erential Equations, SIAM, Philadelphia, 1992.

[12] K. Michael, Fast iterative methods for symmetric Sinc-Galerkin system, IMA J. Numer. Anal., 19 (1999), pp. $357-373$.

[13] W. C. H. Slemp and R. K. Kapania, Imposing boundary conditions in Sinc method using highest derivative approximation, Journal of Computational and Applied Mathematics, 230 (2009) pp. 371-392.

[14] F. Stenger, Numerical Methods Based on Sinc and Analytic Functions, Springer-Verlag, New York, 1993.

[15] F. Stenger, A SincGalerkin method of solution of boundary value problems, Math. Comp., 33 (1979), pp. 85-109.

[16] F. Stenger, Summary of Sinc numerical methods, Journal of Computational and Applied Mathematics, 121 (2000), pp. 379-420.

[17] D. Trif, On sinc methods for partial diff erenial equation, Seminar on Fixed Point Theory Cluj-Napoca, Volume 3, (2002), pp.173-182.

[18] Ali Salimi Shamloo, Sanam Shahkar, Alieh Madadi, Numerical Solution of the Fredholme-Volterra Integral Equation by the Sinc Function, American Journal of Computational Mathematics, 2012, 2, 136-142. 